\title{
The relationship between physicians and Pharma
}

Playing the devil's advocate

John R. Corboy, MD, FAAN

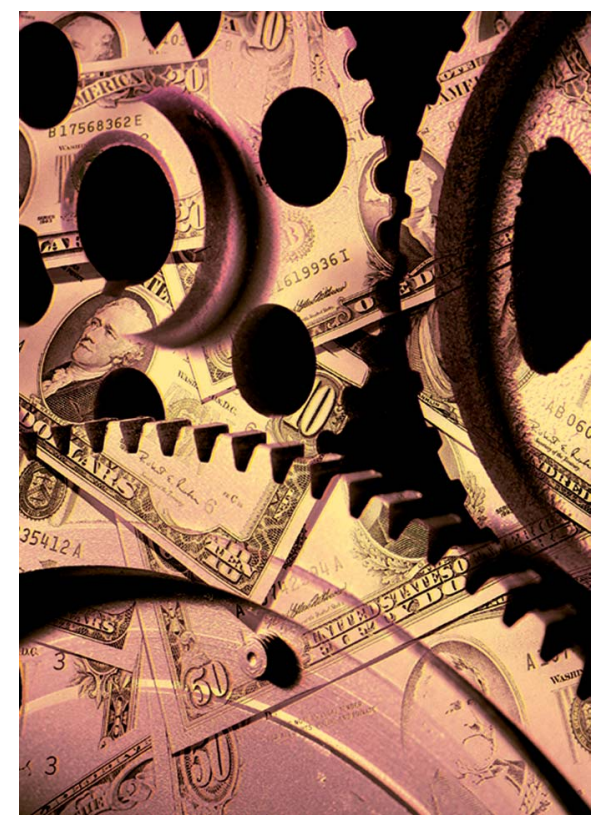

A

11 who practice medicine face, on a daily basis, conflicts of interest (COIs) that have the potential to affect patient care. COIs may exist within our own practice. We may choose to perform (or not perform) a procedure that may (or may not) benefit the patient but clearly brings income to the practice; we may see patients in the office for services that might just as easily be taken care of by phone.

There may be third parties with whom we interact — either inside or outside the office-who benefit from their interaction with us, but with uncertain effects on our patients or the cost of medicine. The most overt interactions include those with representatives of pharmaceutical companies (hereafter Pharma), everything from speaking to a "drug rep" to accepting modest gifts, accepting lunches for office staff, speaking as part of a "speakers' bureau," participating in clinical trials, consulting, or other more formal relationships.

Other activities that are associated with potential COIs include participating in the medicolegal system, and advising investor groups, such as fund managers, about the potential investment of millions of dollars. In all of these circumstances, there are "terms of engagement," but all are fraught with potential and real COIs. 


\section{It is extremely unlikely the federal government or other entities could simply replace all the clinical research done by Pharma.}

There are a variety of mechanisms in place to limit, mitigate, or avert COIs. The first principle is making full and formal disclosure of the relationships that might lead to COIs. It remains to be seen if the Sunshine Act affects attendance at industry-sponsored events, or if our patients make decisions about providers based on reported financial remuneration from industry. The second principle is managing COIs in the light of day without compromising professional and personal ethics. The third is avoiding or withdrawing from activities in which the COIs cannot be managed.

In the interest of exploring our readers' views on these matters, we attempted to design a procon set of articles with divergent views. It became clear when we attempted to enlist someone to argue the pro side of this controversy that the relationship between physicians and Pharma was, is, and will forever be, an extremely touchy subject. Some potential authors politely declined the invitation, citing time constraints. One declined, but boldly exposed the 700-pound gorilla in the room: "To write this is a no-win situation for the author and will leave s/he open to criticism." Thus, the Editor has taken up the gauntlet (or hot potato?) and passes it quickly on to the reader.

In this issue, Dr. Carl Elliot reasserts examples in which the interactions between physicians and Pharma have gone terribly wrong, and argues that all relationships between Pharma and physicians have an inherent flaw that exposes physicians to intractable COIs, with potentially negative outcomes for patients. While I think we can all agree that accepting money or favors under a variety of circumstances that have little or no benefit to the patient, or may harm the patient, are inherently unethical, there are likely 2 sides to this discussion. While Dr. Elliot correctly and appropriately focuses on potential abuses within the relationships between physicians and Pharma, there are likely benefits as well.

In the interest of presenting the other side of the argument, we offer some arguably positive aspects of the relationship between physicians and Pharma for our readers' consideration. To stimulate discussion, we have started a list of pros, i.e., reasons why physicians should consider having relationships with Pharma.

First, there may be benefits to society. Clinical research is going to get done, one way or the other. It is important for both academic physicians and those in practice to participate, so as to make sure the companies are doing the right studies, the right way. While no one would suggest that Pharma decides to do studies only with guidance from outside physicians, it is certainly true that physician consultants help shape the type and nature of studies that get performed. If there is no input from outside Pharma, who will be protecting the rest of society? In a similar fashion, while it is theoretically possible for physicians to become shills for plaintiff or defense attorneys willing to pay for their opinions, is it not important, given that the tort system in the United States is unlikely to simply disappear overnight, that all of us consider offering honest, uncoached opinions in the name of reaching of an appropriate result within the legal system? If not us, then whom? Another weighty consideration for clinical research is that, practically speaking, Pharma has no access to patients, nor does it currently have the apparatus to collect them. No research $=$ no progress. It is extremely unlikely the federal government or other entities could simply replace all the clinical research done by Pharma.

Second, there may be indirect benefits to patients from physicians interacting with Pharma. For example, physicians may sit on data safety monitoring boards, providing unique skill sets that protect patients by shining the light on adverse events of drugs in clinical trials, or may even close trials completely.

Third, patients may directly benefit from the physician-Pharma relationships. Regarding research, they may gain access to medications or devices at a much earlier time in their illness. 


\section{Are physicians, and the systems available to them, able to adequately manage potential COls or not? Is full disclosure adequate?}

This may be especially important for those who have failed one or more approaches in the past. In interactions with medical science liaisons, the physician may learn valuable details about mechanism of actions of drugs, or drug-drug interactions, which might suggest using, or not using, a particular medication.

The related questions have to do with managing potential COIs. Here, there are many questions. Are physicians, and the systems available to them, able to adequately manage potential COIs or not? Is full disclosure adequate? Will further limitations or systems responses be adequate? Could we do more? Or, is the nature of the potential conflict, and any potential response, by its very nature, inadequate to sufficiently protect society from the potential negative outcomes of these interactions?

And so, we ask our readers to weigh in. Based on the nature of the responses, we will either request a response from Dr. Elliot, or respond ourselves. We reserve the right to combine, condense, or otherwise compile the responses in a manner designed to maximize diversity in the responses. We want your input and thoughts.

\section{STUDY FUNDING}

No targeted funding reported.

\section{DISCLOSURES}

J. Corboy served as a Section Editor for Neurology ${ }^{\circledR}$ Today and serves as editor of Neurology ${ }^{\circledR}$ Clinical Practice; serves on the speakers' bureau for ProCE; receives/received research support from Genentech, Novartis, Biogen Idec, Teva, Eli Lilly, Orasi Medical, NIH, Juvenile Diabetes Research Foundation, and National MS Society; and has participated in medico-legal cases. Full disclosure form information provided by the author is available with the full text of this article at Neurology.org/cp.

\section{Related articles from other AAN physician and patient resources}

\section{Neurology ${ }^{\circledR} \bullet$ Neurology.org}

Restoring medical professionalism

August 21, 2012;79:820-827.

American Academy of Neurology policy on pharmaceutical and device industry support March 6, 2012;78:750-754. 